\title{
INTEGRAL EQUATION METHODS IN INVERSE OBSTACLE SCATTERING
}

\author{
RAINER KRESS ${ }^{1}$
}

(Received 16 March 1998)

\begin{abstract}
In this survey we consider a regularized Newton method for the approximate solution of the inverse problem to determine the shape of an obstacle from a knowledge of the far field pattern for the scattering of time-harmonic acoustic or electromagnetic plane waves. Our analysis is in two dimensions and the numerical scheme is based on the solution of boundary integral equations by a Nyström method. We include an example of the reconstruction of a planar domain with a corner both to illustrate the feasibility of the use of radial basis functions for the reconstruction of boundary curves with local features and to connect the presentation to some of the research work of Professor David Elliott.
\end{abstract}

\section{The inverse obstacle problem}

The purpose of this survey is to introduce the reader to inverse obstacle scattering problems for time-harmonic waves by considering the case of two dimensions, that is, scattering of time-harmonic acoustic or electromagnetic waves by infinitely long cylindrical obstacles. Let us denote the cross section of the cylindrical obstacle by $D$ and assume that $D \subset \mathbb{R}^{2}$ is a bounded and simply connected domain with boundary $\partial D$ of class $C^{2}$. The simplest direct obstacle scattering problem for time-harmonic waves is, given an incident field $u^{i}$ as an entire solution of the Helmholtz equation, to find the total field $u=u^{i}+u^{s}$ such that $u$ satisfies the Helmholtz equation

$$
\Delta u+k^{2} u=0
$$

with wave number $k>0$ in the exterior $\mathbb{R}^{2} \backslash \bar{D}$ of $D$, the Dirichlet boundary condition

$$
u=0 \text { on } \partial D
$$

\footnotetext{
'Institut für Numerische und Angewandte Mathematik, Universität Göttingen, Lotzestr. 16-18, D-37083 Göttingen, Germany.

(C) Australian Mathematical Society 2000 , Serial-fee code $0334-2700 / 00$
} 
and the Sommerfeld radiation condition

$$
\lim _{r \rightarrow \infty} \sqrt{r}\left(\frac{\partial u^{s}}{\partial r}-i k u^{s}\right)=0, \quad r=|x|,
$$

uniformly for all directions. In acoustics the Dirichlet condition corresponds to scattering from a sound-soft obstacle whereas in electromagnetics it models scattering from a perfect conductor with the electromagnetic field $E$-polarized parallel to the axis of the cylinder.

The Sommerfeld radiation condition ensures uniqueness of the solution to the scattering problem (1.1)-(1.3). Existence of a solution was established through boundary integral equation methods by Vekua, Weyl and Müller in the 1950s. In particular, if the incident field $u^{i}$ is an entire solution to the Helmholtz equation, then there exists a unique solution $u \in C^{2}\left(\mathbb{R}^{2} \backslash \bar{D}\right) \cap C^{1, \alpha}\left(\mathbb{R}^{2} \backslash D\right)$ for $0<\alpha<1$, that is, a solution which is Hölder continuously differentiable up to the boundary. For details on uniqueness and existence we refer to $[3,4]$ and also to Section 4.

For the scattered wave, the Sommerfeld radiation condition (1.3) implies an asymptotic behavior in the form of an outgoing cylindrical wave

$$
u^{s}(x)=\frac{e^{i k|x|}}{\sqrt{|x|}}\left\{u_{\infty}\left(\frac{x}{|x|}\right)+O\left(\frac{1}{|x|}\right)\right\}, \quad|x| \rightarrow \infty .
$$

The function $u_{\infty}$, defined on the unit circle $\Omega:=\left\{z \in \mathbb{R}^{2}:|z|=1\right\}$, is known as the far field pattern or scattering amplitude of the scattered wave $u^{s}$. The inverse problem we are concerned with is, given the far field pattern $u_{\infty}$ of the scattered wave $u^{s}$ for one incoming plane wave $u^{i}(x)=e^{i k x \cdot d}$ with incident direction $d \in \Omega$, to determine the shape of the scatterer $D$. This is a model problem for applications in radar, sonar, geophysical exploration, medical imaging and nondestructive testing and is difficult to solve since it is nonlinear and improperly posed. It is nonlinear since the solution to the direct scattering problem depends nonlinearly on the boundary $\partial D$, and it is improperly posed since the construction of the scattered wave $u^{s}$ from its far field pattern $u_{\infty}$ is severely improperly posed. Of particular interest is the inverse obstacle scattering problem for frequencies in the resonance region, that is, for scatterers $D$ and wave numbers $k$ such that the wavelengths $2 \pi / k$ are of a comparable size to the diameter of the scatterer. In this intermediate frequency range, low frequency methods such as impedance tomography or high frequency methods such as physical or geometrical optics do not yield valid approximations. For a detailed study of this inverse obstacle scattering problem we refer to [4].

Before we proceed we note that besides the Dirichlet condition (1.2) other boundary conditions need to be allowed and considered. For example, the Neumann condition models the scattering from a sound-hard obstacle by an acoustic wave or the scattering from a perfect conductor by an $H$-polarized electromagnetic wave. The scattering 
from penetrable obstacles is described by transmission conditions or by conductive and resistive boundary conditions.

\section{Uniqueness}

The first question to ask about the inverse obstacle scattering problem is that of uniqueness. This is of importance both for theoretical study and for the implementation of numerical algorithms. The following result is due to Schiffer.

THEOREM 1. Assume that $D_{1}$ and $D_{2}$ are two sound-soft scatterers such that the far field patterns coincide for an infinite number of plane waves with distinct incident directions and one fuxed wave number. Then $D_{1}=D_{2}$.

For proving this uniqueness result and corresponding results for other boundary conditions, in principle, two different approaches can be used. The first method goes back to Schiffer and is restricted to the case of the Dirichlet boundary condition. It uses the fact that the eigenspaces of the Laplacian in bounded domains $B$ with Dirichlet boundary condition have finite dimension regardless of the smoothness of the boundary $\partial B$. Given two different scatterers $D_{1}$ and $D_{2}$, this property is used for the difference set $B=\left(\mathbb{R}^{2} \backslash \bar{G}\right) \backslash \bar{D}_{2}$, where $G$ is the unbounded component of the complement of $\bar{D}_{1} \cup \bar{D}_{2}$. Even for smooth $\partial D_{1}$ and $\partial D_{2}$ the set $B$ can have a very irregular boundary, and therefore Schiffer's argument cannot be immediately transferred to other boundary conditions such as the Neumann boundary condition where the finiteness of the dimension of the eigenspaces requires some regularity of the boundary.

A variational approach to proving uniqueness for the inverse transmission problem was introduced by Isakov [13] and was simplified and also applied to the Neumann boundary condition by Kirsch and Kress [18]. In principle, the uniqueness proof in [18] (see also [4]) proceeds in two steps: first, from the coincidence of the far field patterns for two obstacles $D_{1}$ and $D_{2}$ for all incident plane waves it is deduced that the far field patterns and hence the scattered waves also coincide for point sources as incident fields with the source located in the domain $G$ as defined above. Then in the second step, assuming that $D_{1} \neq D_{2}$, a contradiction is obtained by letting the point sources tend to a point $x^{*}$ which is a boundary point of one of the obstacles but not of the other. Assume without loss of generality that $x^{*} \in \partial D_{1}$ and $x^{*} \notin \partial D_{2}$. Then the well-posedness of the exterior boundary value problem with respect to the boundary $\partial D_{2}$ ensures that the scattered wave remains bounded (in a neighborhood of $x^{*}$ ) as the incident source point approaches $x^{*}$. On the other hand, by the boundary condition with respect to the boundary $\partial D_{1}$, the scattered wave cannot remain bounded in $x^{*}$ when the incident source points tend to $x^{*}$. 
The methods of Isakov and of Kirsch and Kress have been extended to establish uniqueness for the inverse conductive and resistive boundary value problem by Hettlich [11] and by Gerlach and Kress [9]. They have also been used and appropriately modified by Colton, Kress and Monk [5] to prove uniqueness for the inverse electromagnetic scattering problem from a homogeneous orthotropic medium. Potthast [33] has developed a related reconstruction algorithm based on the idea of using point sources.

Employing the monotonicity property of the eigenvalues of the Laplacian for the Dirichlet boundary condition, Colton and Sleeman [6] have shown that if it is known that the scatterer $D$ is contained in a disk of radius $R$ such that $k R$ is smaller than the smallest positive zero $\zeta_{0}=2.404 \ldots$ of the Bessel function $J_{0}$ of order zero, then the far field pattern for one incident wave uniquely determines $D$ (see also [4, Corollary 5.3]). It remains a challenging open problem to establish uniqueness for one incident plane wave without assuming any a priori information.

\section{Reconstruction methods}

Given the success of integral equation methods for the solution of the direct obstacle scattering problem, it is not surprising that integral equations also play an important role in the approximate solution of the inverse scattering problem. In principle, two different approaches for the approximate solution of the inverse problem can be used. In the first group of methods the inverse obstacle problem is separated into the linear ill-posed part for the reconstruction of the scattered wave $u^{s}$ from its far field pattern $u_{\infty}$ and the nonlinear well-posed part for finding the location of the boundary $\partial D$ of the scatterer from the boundary condition (1.2) for the total field $u^{i}+u^{s}$. For an extensive study of typical examples for this approach we refer the reader to [4]. In the second group of methods the inverse obstacle problem is considered as an ill-posed nonlinear operator equation. Each algorithm of this second group requires the solution of the direct scattering problem at each step of the iteration method used to arrive at an approximate solution. Hence until a few years ago these methods seemed to be too computationally costly to be competitive with the first group of methods and therefore research concentrated mainly on the first group. However, with the advances being made in computers, computing time becomes a less important issue and therefore recently the application of methods such as regularized Newton iterations to inverse obstacle scattering has been more intensively investigated. Therefore, in this survey, we will outline some of the analysis required for the implementation of Newton-type methods in inverse obstacle scattering. For a more extensive survey we refer the reader to [25].

For a fixed incident field $u^{i}$, the solution to the direct scattering problem defines 
an operator $F: \partial D \mapsto u_{\infty}$ which maps the boundary $\partial D$ of the scatterer $D$ onto the far field pattern $u_{\infty}$. In terms of this operator, given a far field pattern $u_{\infty}$, the inverse problem consists of solving the equation

$$
F(\partial D)=u_{\infty}
$$

for the unknown boundary $\partial D$. A parametrization of the boundary curve is needed both for the theoretical investigation and the numerical solution of (3.1). For simplicity, we assume the scatterer to be starlike with respect to the origin. However, we note that the following analysis can be extended to a wider class of boundary representations. We parametrize

$$
\partial D=\{r(z) z: z \in \Omega\}
$$

with some function $r \in C_{+}^{2}(\Omega)$ where $C_{+}^{2}(\Omega)$ denotes the cone of twice continuously differentiable functions $r: \Omega \rightarrow(0, \infty)$. Then we can view $F$ as a nonlinear operator from $C_{+}^{2}(\Omega)$ into $L^{2}(\Omega)$ and will write $F(r)$ instead of $F(\partial D)$. The choice of the image space $L^{2}(\Omega)$ is dictated by its suitability to describe errors in the measured far field data.

We now summarize the basic properties of the operator $F$.

THEOREM 2. The far field operator $F: C_{+}^{2}(\Omega) \rightarrow L^{2}(\Omega)$ is injective on the set $\left\{r \in C_{+}^{2}(\Omega): k\|r\|_{\infty}<\zeta_{0}\right\}$, where $\zeta_{0}=2.404 \ldots$ denotes the smallest positive zero of the Bessel function $J_{0}$ of order zero.

This is a reformulation of the uniqueness result of Colton and Sleeman mentioned above.

THEOREM 3. The far field operator $F: C_{+}^{2}(\Omega) \rightarrow L^{2}(\Omega)$ is continuous and compact.

This theorem indicates the ill-posedness of the inverse scattering problem. For a proof we refer to [4, Theorem 5.9].

THEOREM 4. The far field operator $F: C_{+}^{2}(\Omega) \rightarrow L^{2}(\Omega)$ is Fréchet differentiable. The derivative is given by

$$
F^{\prime}(r) h=v_{\infty}
$$

where $v_{\infty}$ is the far field pattern of the solution $v$ to the Helmholtz equation

$$
\Delta v+k^{2} v=0
$$


in $\mathbb{R}^{2} \backslash \bar{D}$ satisfying the Sommerfeld radiation condition and the Dirichlet boundary condition

$$
v=-v \cdot \tilde{h} \frac{\partial u}{\partial v} \text { on } \partial D
$$

where $\tilde{h}(r(z))=h(z) z, z \in \Omega$, and $v$ denotes the outward unit normal to $\partial D$.

For a proof via Hilbert space methods we refer to [4, Theorem 5.7] and to Kirsch [16]. Proofs by boundary integral equation methods were first given by Potthast $[30,31]$ and can also be found in $[21,22]$. The boundary condition (3.2) can be formally obtained by differentiating the boundary condition (1.2) with respect to $r$ and, of course, is related to Hadamard's classical variational formula in fluid dynamics from 1912. The analysis on the differentiability with respect to the boundary has also been extended to other boundary conditions with appropriate changes in the resulting boundary condition for the derivative (see [29, 32]).

THEOREM 5. The Fréchet derivative $F^{\prime}(r): L^{2}(\Omega) \rightarrow L^{2}(\Omega)$ is injective and has dense range.

These properties of the derivative are of relevance for the application of regularization techniques to the linearized version of (3.1). For proofs we refer to $[15,26]$ and note that the injectivity is a consequence of the boundary condition (3.2) and Holmgren's uniqueness theorem. Since $F^{\prime}(r) h$ is a far field pattern, the Fréchet derivative represents a compact operator $F^{\prime}(r): L^{2}(\Omega) \rightarrow L^{2}(\Omega)$.

In Newton's method, in the usual fashion, the nonlinear equation (3.1) is replaced by the linearized equation

$$
F(r)+F^{\prime}(r) h=u_{\infty},
$$

which has to be solved for $h$ in order to improve an approximate boundary curve given by the radial function $r$ into the new approximation given by $\tilde{r}=r+h$. Then Newton's method consists of iterating this procedure. However, since $F^{\prime}(r)$ is compact, the linear equation (3.3) is ill-posed. Therefore regularization techniques such as Tikhonov regularization or singular value cut-off have to be applied. Using Tikhonov regularization, the ill-posed equation (3.3) is stabilized through replacing it by the penalized equation

$$
\alpha h+\left[F^{\prime}(r)\right]^{*} F^{\prime}(r) h=\left[F^{\prime}(r)\right]^{*}\left\{u_{\infty}-F(r)\right\},
$$

where $\left[F^{\prime}\left(r_{n}\right)\right]^{*}$ denotes the $L^{2}$ adjoint of $F^{\prime}(r)$ and $\alpha>0$ is a small regularization parameter. In practice, the parameter $\alpha$ is mostly chosen either by a discrepancy principle or by trial and error. 
For an analysis on how to adjust the choice of the regularization parameter to the number of iteration steps and for the convergence of regularized Newton schemes for general ill-posed nonlinear operator equations and possible applications to inverse obstacle scattering we refer to $[2,12]$.

In practical computations $h$ is taken from an appropriately chosen finite dimensional subspace $W_{N} \subset C^{2}(\Omega)$ with dimension $N$ and (3.3) is approximately solved by collocation at $M$ equidistantly spaced points $z_{1}, \ldots, z_{M} \in \Omega$. Then writing

$$
h=\sum_{j=1}^{N} a_{j} h_{j}
$$

where $h_{1}, \ldots, h_{N}$ denotes a basis of $W_{N}$, the linear system

$$
\sum_{j=1}^{N} a_{j}\left(F^{\prime}(r) h_{j}\right)\left(z_{i}\right)=u_{\infty}\left(z_{i}\right)-F(r)\left(z_{i}\right), \quad i=1, \ldots, M,
$$

has to be solved for the coefficients $a_{1}, \ldots, a_{N} \in \mathbb{R}$ by Tikhonov regularization, that is, by a penalized least squares method.

In order to set up the linear system (3.5) computationally, in each iteration step the direct scattering problem for the boundary $\partial D$ given by the radial function $r$ has to be approximately solved for the evaluation of $F(r)\left(z_{i}\right)$ and to obtain the normal derivative $\partial u / \partial v$ of the total field $u$ which enters the boundary condition (3.2) for the Fréchet derivative. In addition, for the matrix entries $\left(F^{\prime}(r) h_{j}\right)\left(z_{i}\right)$ one has to solve $N$ Dirichlet problems for the same boundary $\partial D$ and different boundary values given by (3.2) for the basis functions $h=h_{j}, j=1, \ldots, N$. In principle, these computations can be done by any numerical method for solving the exterior Dirichlet problem for the Helmholtz equation. However, we recommend using a boundary integral equation approach as described in [4]. We will comment on this in the following section.

For more details on the numerical implementation of the above method and for numerical examples the reader is referred to [17, 21, 22]. The Newton method has also been extended and successfully tested for other boundary conditions [29] and for scattering from cracks $[23,24,28]$. All these numerical examples include testing the stability of the method with respect to erroneous far field data. The numerical performance indicates that the accuracy of the reconstructions obtained by the regularized Newton method is superior to those obtained by methods of the first group mentioned at the beginning of this section.

Most of the numerical reconstructions mentioned above use trigonometric polynomials for the approximating space $W_{N}$. For a more accurate reconstruction of details of the boundary curve it might be advantageous to replace the global trigonometric functions by functions with a more local structure. In order to illustrate this, we 
choose a radial basis function in the form of a periodic Gaussian function

$$
g(t):=\exp \left(-\gamma \sin ^{2} \frac{t}{2}\right)
$$

with some $\gamma>0$ and use approximations

$$
h(t)=\sum_{j=1}^{N} a_{j} g\left(N\left(t-t_{j}\right)\right),
$$

where $t_{j}=2 \pi j / N$.

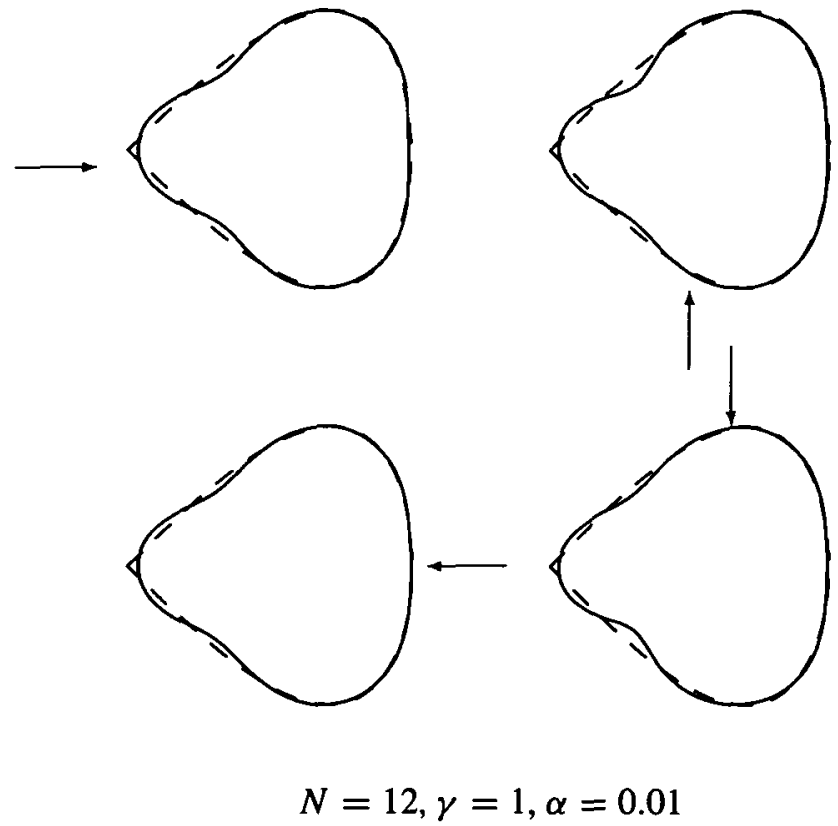

FiGURE 1. Reconstruction of a drop-shaped domain for $k=1$

For the following numerical example the boundary curve actually has a corner and is given by the parametric representation

$$
x(t)=\left(2 \sin \frac{t}{2},-\sin t\right), \quad 0 \leq t \leq 2 \pi,
$$

which describes a drop-shaped starlike curve. For the solution of the boundary integral equations in each iteration step we used the Nyström method on an equidistant mesh with 32 grid points. In order to avoid committing an inverse crime (see [4, p. 133]), for generating the synthetic data $u_{\infty}$ for the exact boundary curve (3.7) with a corner, we 
used a graded mesh with 128 grid points and a different coupling parameter $\eta$ (see (4.2) and (4.4)). As an initial guess for the Newton iteration we chose the unit circle. As a stopping rule for the number of iterations we used the residual $R:=\left\|F(r)-u_{\infty}\right\|_{L^{2}(\Omega)}$ and terminated the iterations when the difference of the value of $R$ for two consecutive iterations was less than a tolerance value $\delta$, say $\delta=10^{-4}$.

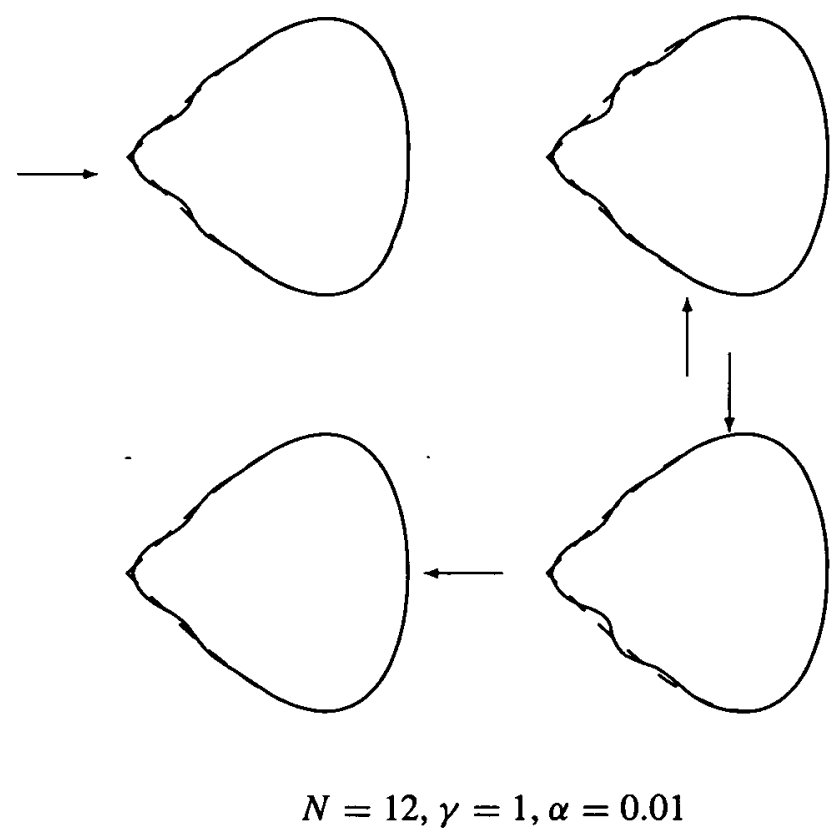

FIGURE 2. Reconstruction of a drop-shaped domain for $k=3$

In the figures above, the dashed lines give the exact boundary curve and the full lines give the reconstructions. The arrows indicate the incident directions $d$. The figures confirm that the basis functions (3.6) are suited to reconstruct the shape of the corner despite the fact that they do not allow the exact representation of the corner. The figures illustrate that increasing the wave number, that is, decreasing the wave length leads to a better recovery of details of the scatterer at the possible expense of poorer reconstructions in the shadow region.

\section{Boundary integral equations}

We will conclude with some remarks on the solution of the direct boundary value problems required for the computation of the right-hand sides $F(r)\left(z_{i}\right)$ and the matrix elements $\left(F^{\prime}(r) h_{j}\right)\left(z_{i}\right)$. The fundamental solution to the Helmholtz equation in two 
dimensions is given by

$$
\Phi(x, y):=\frac{i}{4} H_{0}^{(1)}(k|x-y|), \quad x \neq y,
$$

where $H_{0}^{(1)}$ is the Hankel function of order zero and of the first kind. Recall that by $v$ we denote the outward unit normal to $\partial D$. The classical approach to solving the exterior Dirichlet problem is to seek the solution in the form of a combined acoustic double- and single-layer potential

$$
v(x)=\int_{\partial D}\left\{\frac{\partial \Phi(x, y)}{\partial \nu(y)}-i \eta \Phi(x, y)\right\} \varphi(y) d s(y), \quad x \in \mathbb{R}^{2} \backslash \bar{D},
$$

with some positive coupling parameter $\eta$. Then from the potential theoretic jump relations ( $c f$. [3]) it follows that (4.1) solves the Dirichlet problem with boundary condition $v=f$ provided the density $\varphi$ is a solution of the integral equation of the second kind

$$
\varphi+K \varphi-i \eta S \varphi=2 f,
$$

where $K, S: C(\partial D) \rightarrow C(\partial D)$ denote the integral operators defined by

$$
(K \varphi)(x):=2 \int_{\partial D} \frac{\partial \Phi(x, y)}{\partial \nu(y)} \varphi(y) d s(y), \quad x \in \partial D,
$$

and

$$
(S \varphi)(x):=2 \int_{\partial D} \Phi(x, y) \varphi(y) d s(y), \quad x \in \partial D .
$$

These integral operators are compact since they have weakly singular kernels. Therefore the existence of a solution can be established by the Riesz-Fredholm theory for compact operators by showing that the homogeneous form of (4.2) admits only the trivial solution.

In addition to transforming the direct scattering problem into integral equations by the above layer approach (for the scattered wave $u^{s}$ with boundary condition $u^{s}=-u^{i}$ on $\partial D$ ) it is also possible to obtain integral equations using the so-called direct method. From Green's formula for solutions to the Helmholtz equation it can be deduced that for the scattering of an entire field $u^{i}$ from a sound-soft obstacle $D$ we have the representation

$$
u(x)=u^{i}(x)-\int_{\partial D} \Phi(x, y) \frac{\partial u}{\partial v}(y) d s(y), \quad x \in \mathbb{R}^{2} \backslash \bar{D},
$$

of the total field $u$ in terms of its normal derivative on the boundary (see $[4$, p. 53]). From this, again with the aid of the jump relations and the boundary condition $u=$ 
$u^{i}+u^{s}=0$ on $\partial D$, it can be seen that the normal derivative $\psi=\partial u / \partial v$ satisfies the integral equation

$$
\psi+K^{\prime} \psi-i \eta S \psi=2 \frac{\partial u^{i}}{\partial \nu}-2 i \eta u^{i}
$$

where the integral operator $K^{\prime}: C(\partial D) \rightarrow C(\partial D)$ given by

$$
\left(K^{\prime} \psi\right)(x):=2 \int_{\partial D} \frac{\partial \Phi(x, y)}{\partial \nu(x)} \psi(y) d s(y), \quad x \in \partial D,
$$

is the adjoint of $K$. Since the integral equation (4.4) is adjoint to (4.2), by the Riesz-Fredholm theory, again it is uniquely solvable.

Using (4.4) has the advantage of yielding immediately approximate values for the normal derivative of the total field, which enters the boundary condition (3.2) for the Fréchet derivatives. For the solution of the boundary value problem (3.2) we suggest using the integral equation (4.2) (with the right-hand side $f=-v \cdot \tilde{h}_{j} \partial u / \partial v$ ). If we used the integral equation derived from the Green's representation formula here we would be faced with the problem that the corresponding right-hand sides require the numerical computation of the normal derivative of a combined double- and singlelayer potential (see [3, p. 103]). Note that the same problem would come up in the computation of the boundary values in (3.2) if we used (4.2) instead of (4.4) for the evaluation of $F(z)\left(\hat{x}_{i}\right)$. To avoid the need to set up the matrix for the numerical evaluation of the normal derivative of the combined double- and single-layer potential, we think it preferable to use two adjoint integral equations which simply lead to two transposed matrices in the approximating linear systems. Hence the solution of the second linear system which has to be done simultaneously for $N$ different right-hand sides corresponding to setting $h=h_{j}, j=1, \ldots, N$, can be performed cheaply by an $L R$-decomposition for the matrix of the first system.

Using a parametric representation $\partial D=\{x(t): 0 \leq t \leq 2 \pi\}$, where it is tacitly assumed that the parameter $t$ is equivalent to arc length on the boundary in the sense that there exist positive constants $c_{1}$ and $c_{2}$ such that $c_{1} t \leq s \leq c_{2} t$, both integral equations (4.2) and (4.4) can be brought into a form

$$
\chi(t)-\int_{0}^{2 \pi} K(t, \tau) \chi(\tau) d \tau=g(t), \quad 0 \leq t \leq 2 \pi,
$$

with the kernel $K$, the right-hand side $g$ and the solution $\chi$ appropriately defined. Since the Hankel function $H_{0}^{(1)}$ has a logarithmic singularity, for both (4.2) and (4.4) the kernel $K$ can be decomposed in the form

$$
K(t, \tau)=K_{1}(t, \tau) \ln \left(4 \sin ^{2} \frac{t-\tau}{2}\right)+K_{2}(t, \tau)
$$


with smooth functions $K_{1}$ and $K_{2}$ provided the boundary $\partial D$ is smooth. For the numerical solution of integral equations of this type a Nyström method using trigonometric interpolation quadratures on an equidistant grid (on the parameter interval $[0,2 \pi]$ ) is most effective. For details on the numerical implementation and the corresponding error analysis we refer to $[4,19]$.

In particular, if the boundary curve is analytic this Nyström method for the obstacle scattering problem converges exponentially when the number of grid points is increased, that is, the number of correct digits in the approximate solution is doubled if the number of grid points is doubled. In our example we used this method in each Newton step since the boundaries generated by the shape functions (3.6) are analytic.

For domains with corners, however, due to the singularity of the solution at the corner a quadrature method based on an equidistant grid yields only very poor convergence. Therefore, in order to increase the numerical efficiency, the equidistant mesh has to be replaced by a graded mesh. The simplest way to create a grading is to substitute new variables

$$
t=w(s), \quad \tau=w(\sigma),
$$

where the function $w:[0,2 \pi] \rightarrow[0,2 \pi]$ is bijective, strictly monotonically increasing and infinitely differentiable such that the derivatives of $w$ at the end points of the interval $[0,2 \pi]$ vanish up to a certain order $p$. Because of their $S$-shape, David Elliott [7] suggested calling functions of this type sigmoidal functions. After substituting (4.6) for the transformed integral equation in the variables $s$ and $\sigma$, the Nyström method on a equidistant grid can be applied and leads to a rapid convergence provided the order $p$ is chosen appropriately. For details on the numerical implementation and for an error analysis we refer to $[4,20]$. In our example we used this method for generating the synthetic data $u_{\infty}$ for the exact drop-shaped boundary curve (3.7). Related Nyström methods on a graded mesh have been considered and investigated in $[1,10,14,27]$.

The kernel of the double-layer integral operator $K$ in a domain with a corner has a Mellin-type singularity at the corner and is no longer compact in the space of continuous functions. Hence an appropriate general tool to analyze the convergence and the error occurring in the Nyström method on a graded mesh for the double-layer potential operator is the Mellin transform. This has been successfully demonstrated by Elliott and Prössdorf [8].

\section{References}

[1] K. E. Atkinson and I. G. Graham, "An iterative variant of the Nyström method for boundary integral equations on non smooth boundaries", in The Mathematics of Finite Elements and Applications VI (MAFELAP 1987) (ed. Whiteman), (Academic Press, London, 1988) 197-304. 
[2] B. Blaschke, A. Neubauer and O. Scherzer, "On convergence rates for the iteratively regularized Gauss-Newton method", IMA J. Numerical Anal. 17 (1997) 421-436.

[3] D. Colton and R. Kress, Integral Equation Methods in Scattering Theory (Wiley-Interscience Publication, New York, 1983).

[4] . D. Colton and R. Kress, Inverse Acoustic and Electromagnetic Scattering Theory 2nd edition (Springer, Berlin, 1998).

[5] D. Colton, R. Kress and P. Monk, "Inverse scattering from an orthotropic medium", J. Comp. Appl. Math. 81 (1997) 269-298.

[6] D. Colton and B. D. Sleeman, "Uniqueness theorems for the inverse problem of acoustic scattering", IMA J. Appl. Math. 31 (1983) 253-259.

[7] D. Elliott, "The cruciform crack problem and sigmoidal transformations", Math. Meth. in the Appl. Sci. 20 (1997) 121-132.

[8] D. Elliott and S. Prössdorf, "An algorithm for the approximate solution of integral equations of Mellin type", Numer. Math. 70 (1995) 427-452.

[9] T. Gerlach and R. Kress, "Uniqueness in inverse obstacle scattering with conductive boundary condition", Inverse Problems 12 (1996) 619-625.

[10] I. G. Graham and G. A. Chandler, "High-order methods for linear functionals of solutions of second kind integral equations", SIAM J. Numer. Anal. 25 (1988) 1118-1173.

[11] F. Hettlich, "On the uniqueness of the inverse conductive scattering problem for the Helmholtz equation", Inverse Problems 10 (1994) 129-144.

[12] T. Hohage, "Logarithmic convergence rates of the iteratively regularized Gauss-Newton method for an inverse potential and an inverse scattering problem", Inverse Problems 13 (1997) 1279-1 299.

[13] V. Isakov, "On uniqueness in the inverse transmission scattering problem", Comm. Part. Diff. Equa. 15 (1990) 1565-1587.

[14] Y. Jeon, "A Nyström method for boundary integral equations in domains with a piecewise smooth boundary", J. Integral Eqs Appl. 5 (1993) 221-242.

[15] A. Kirsch, "Properties of the far field operators in acoustic scattering", Math. Meth. in the Appl. Sci. 11 (1989) 773-787.

[16] A. Kirsch, "The domain derivative and two applications in inverse scattering theory", Inverse Problems 9 (1993) 81-96.

[17] A. Kirsch, "Numerical algorithms in inverse scattering theory", in Ordinary and Partial Differential Equations, Vol. IV (eds Jarvis and Sleeman), Pitman Research Notes in Mathematics 289, (Longman, London, 1993) 93-111.

[18] A. Kirsch and R. Kress, "Uniqueness in inverse obstacle scattering", Inverse Problems 9 (1993) 285-299.

[19] R. Kress, Linear Integral Equations 2nd edition (Springer, Berlin, 1999).

[20] R. Kress, “A Nyström method for boundary integral equations in domains with corners", Numer. Math. 58 (1990) 145-161.

[21] R. Kress, "A Newton method in inverse obstacle scattering", in Inverse Problems in Engineering Mechanics (eds Bui et al.), (Balkema, Rotterdam, 1994) 425-432.

[22] R. Kress, "Integral equation methods in inverse obstacle scattering", Engineering Anal. with Boundary Elements 15 (1995) 171-179.

[23] R. Kress, "Inverse scattering from an open arc", Math. Meth. in the Appl. Sci. 18 (1995) 267-293.

[24] R. Kress, "Inverse elastic scattering from a crack", Inverse Problems 12 (1996) 667-684.

[25] R. Kress, "Integral equation methods in inverse acoustic and electromagnetic scattering",in Integral Methods in Inverse Analysis (eds Ingham and Wrobel), (Computational Mechanics Publications, Southampton, 1997) 67-92.

[26] R. Kress and W. Rundell, "A quasi-Newton method in inverse obstacle scattering", Inverse Problems 10 (1994) 1145-1157. 
[27] R. Kress, I. N. Sloan and F. Stenger, "A sinc quadrature method for the double-layer integral equation in planar domains with corners", J. Integral Eqs Appl. 10 (1998) 291-316.

[28] L. Mönch, "On the inverse acoustic scattering problem from an open arc: the sound-hard case", Inverse Problems 13 (1997) 1379-1392.

[29] L. Mönch, "A Newton method for solving the inverse scattering problem for a sound-hard obstacle", Inverse Problems 12 (1996) 309-323.

[30] R. Potthast, "Fréchet differentiability of boundary integral operators in inverse acoustic scattering", Inverse Problems 10 (1994) 431-447.

[31] R. Potthast, "Fréchet differentiability of the solution to the acoustic Neumann scattering problem with respect to the domain", J. on Inverse and Ill-posed Problems 4 (1996) 67-84.

[32] R. Potthast, "Domain derivatives in electromagnetic scattering", Math. Meth. in the Appl. Sci. 9 (1996) 1157-1175.

[33] R. Potthast, "A fast new method to solve inverse scattering problems", Inverse Problems 12 (1996) 731-742. 